\title{
Enhancing autophagy by redox regulation extends lifespan in Drosophila
}

3 Authors

4 Helena M. Cochemé ${ }^{1,2,3 *}$, Ivana Bjedov ${ }^{4}$, Sebastian Grönke ${ }^{5}$, Katja E. Menger ${ }^{6}$,

5 Andrew M. James ${ }^{6}$, Jorge Ivan Castillo Quan ${ }^{3}$, Andrea Foley ${ }^{1,2}$, Claudia Lennicke ${ }^{1,2}$,

6 Marcela Buricova ${ }^{1,2}$, Jennifer Adcott ${ }^{3}$, Filipe Cabreiro ${ }^{1,2}$, Michael P. Murphy ${ }^{6}$,

$7 \quad$ Linda Partridge ${ }^{3,5 *}$

8

$9 \quad$ Affiliations

$10{ }^{1}$ MRC London Institute of Medical Sciences, Du Cane Road, London W12 0NN, UK.

$11{ }^{2}$ Institute of Clinical Sciences, Imperial College London, Hammersmith Hospital Campus,

12 Du Cane Road, London W12 0NN, UK.

$13{ }^{3}$ Institute of Healthy Ageing and GEE, University College London, Gower Street, London

14 WC1E 6BT, UK.

$15{ }^{4}$ UCL Cancer Institute, 72 Huntley Street, London WC1E 6DD, UK.

$16{ }^{5}$ Max Planck Institute for Biology of Ageing, Joseph-Stelzmann-Str. 9b, 50931 Cologne,

17 Germany.

$18{ }^{6}$ MRC Mitochondrial Biology Unit, University of Cambridge, Cambridge Biomedical Campus,

19 Hills Road, Cambridge CB2 0XY, UK.

20

21 * correspondence: helena.cocheme@1ms.mrc.ac.uk partridge@age.mpg.de 
Redox signalling is an important modulator of diverse biological pathways and processes, and operates through specific post-translational modification of redox-sensitive thiols on cysteine residues ${ }^{1-4}$. Critically, redox signalling is distinct from irreversible oxidative damage and functions as a reversible 'redox switch' to regulate target proteins. $\mathrm{H}_{2} \mathrm{O}_{2}$ acts as the major effector of redox signalling, both directly and through intracellular thiol redox relays ${ }^{5,6}$. Dysregulation of redox homeostasis has long been implicated in the pathophysiology of many age-related diseases, as well as in the ageing process itself, however the underlying mechanisms remain largely unclear ${ }^{7,8}$. To study redox signalling by $\mathrm{H}_{2} \mathrm{O}_{2}$ in vivo and explore

To explore the role of endogenous redox signalling in vivo, we used the UAS/GAL4 expression system to up-regulate catalase in wild-type (WT) flies. Global up-regulation of catalase under control of the daughterless promoter (da-GAL4>UAS-cat) extended the median and maximum lifespan of female flies (typically by $\sim 10-15 \%$; Fig. 1a). Importantly, for these experiments we used the white Dahomey $\left(w^{D a h}\right)$ background, which is a long-lived and outbred WT, hence we are extending healthy lifespan and not rescuing a short-lived defect. Catalase was overexpressed $\sim 5$-fold at the mRNA level in whole flies (Fig. S1a). Lifespan was not extended in males (Fig. 1a), despite similar catalase over-expression (Fig. S1a), however interventions modulating nutrient-sensing in Drosophila often show gender-specific effects on survival in females, that are absent or marginal in males ${ }^{10}$.

The da-GAL4>UAS-cat flies were mildly delayed in eclosing, without affecting the proportion of larvae surviving to adulthood (Fig. S1b). To exclude developmental effects, we showed that the lifespan extension could be fully recapitulated using the inducible GeneSwitch system, with over-expression from d2 of adulthood onwards (da-GS $>$ UAS-cat \pm RU; Figs. 1b, S1a). 
Varying the dose of the inducer drug RU (50-400 $\mu \mathrm{M})$ still did not extend lifespan in males and had only marginal effects on lifespan extension in females (Fig. S1c), suggesting that the catalase benefits are at maximum potential. Over-expressing catalase under the control of an alternative ubiquitous driver (actin5c-GAL4>UAS-cat) also extended lifespan (Fig. S1d). Furthermore, catalase-mediated lifespan extension was independent of Wolbachia status (Figs. S1e,f), which can influence fly longevity and physiology ${ }^{11,12}$. In addition to lifespan extension, the catalase females also exhibited increased healthspan ${ }^{13}$, as inferred from their enhanced climbing ability with age (Fig. 1c). To explore effects on age-specific mortality, trajectories derived from the survival curves revealed a shift in the intercept, but not the slope (Fig. 1d), indicating that catalase over-expression decreased the overall risk of death, rather than slowing its rate of increase with age ${ }^{14}$. Therefore, the catalase over-expressing flies were healthier for longer.

Using the inducible GeneSwitch system showed that induction of catalase from middle-age (d28 and d42) or old-age (d56) was sufficient to extend lifespan (Figs. 1e, S1g), although not to the full extent from induction at $\mathrm{d} 2$. By d56, the $-\mathrm{RU}$ control flies had already started dying, yet switching to $+R U$ treatment even at this late stage still enhanced survival. This implies that for full benefits the redox shift needs to occur early in life, yet late-onset still offers protection. The level of catalase over-expression induced by RU was equivalent at all ages, as were the levels of endogenous catalase in the controls, eliminating any contribution from changes in RU consumption or endogenous catalase expression with age (Fig. S1h). We conclude that ubiquitous up-regulation of catalase improves healthspan and extends lifespan in female WT flies. Interestingly, tissuespecific catalase up-regulation using a range of drivers (e.g. tubule, gut, fat body, neuronal; Figs. S1i-m), did not recapitulate the strong lifespan extension obtained by the ubiquitous drivers, suggesting that catalase is either acting in an untested tissue (or combination of tissues), or alternatively is required at a more global organismal level.

The catalase over-expressors were exceptionally resistant to multiple modes of oxidative stress - by exogenous $\mathrm{H}_{2} \mathrm{O}_{2}$ (Fig. 1f), the redox cycler paraquat both upon feeding (Fig. 1g) and injection (Fig. 1h), as well as hyperoxia (Figs. 1i, S1n). However, this enhanced oxidative stress resistance is unlikely to explain the lifespan extension in females, because catalase over-expression protected males to a similar extent against oxidative stress without increasing longevity.

To explore the mechanism underlying the catalase-mediated lifespan extension, we examined its relationship to dietary restriction (DR), which is a robust and evolutionary conserved nutritional intervention known to have health and longevity benefits ${ }^{15}$. We measured the lifespan response of catalase over-expressor females to DR by varying the yeast content (i.e. protein source) in the food, while maintaining the sugar content constant ${ }^{16}$. This generated a typical tent-shaped response (Fig. 2a), with lifespan decreased at very low yeast levels $(0.1 \mathrm{x})$, highest under restricted 
conditions $(0.5 \mathrm{x})$, then gradually shortened towards more fully-fed conditions $(1.5 \mathrm{x})$. The lifespan of the catalase flies was enhanced relative to controls at all yeast levels (Fig. 2a), while fecundity increased with yeast content throughout the $0.1-1.5 x$ range for both the control and catalase females (Fig. S2a). Therefore, the catalase over-expressor females exhibit a normal DR response, and the lifespan extension upon catalase up-regulation is not mediated by the activation of DR pathways.

While the DR experiment revealed improved survival compared to control at a range of yeast concentrations (Fig. 2a), including extremely poor nutritional conditions (0.1x-yeast; Fig. 2b), we unexpectedly observed that the catalase over-expressing females, but not males, were acutely sensitive to complete starvation (Fig. 2c). There was no difference in triacylglyceride (TAG) levels at $\mathrm{d} 7(\mathrm{t}=0$ in Fig. 2d) and during a starvation time course (Fig. 2d). Similarly, the levels of glycogen storage and mobilisation were the same in control and over-expressor females (Fig. 2e). Therefore, the starvation sensitivity of the catalase flies was not due to differences in metabolic energy reserves or their mobilisation.

Autophagy is a known longevity assurance process, involved in the response to nutritional challenges such as starvation 17-22. Furthermore, there is evidence for redox-regulation of autophagy ${ }^{23}$. Therefore, we next explored the involvement of autophagy in the differential starvation response and longevity of the catalase flies. To monitor autophagy status in vivo directly, we quantified the levels of Atg8 (LC3 in mammals), a major autophagosome marker. The levels of both the de-lipidated (Atg8-I) and lipidated (Atg8-II) forms were strongly elevated in the catalase over-expressors (Fig. 2f), indicating that autophagy is induced. Indeed, levels of autophagy are physiologically fine-tuned, with both autophagy inhibition and excessive activation shown to induce starvation sensitivity in vivo ${ }^{24,25}$. Furthermore, similarly to the starvation stress, the catalase flies were also sensitive to treatment with the autophagy inhibitor chloroquine (Fig. 2g), confirming that autophagy is affected.

To test the involvement of autophagy in the longevity of the catalase flies, we downregulated autophagy by RNAi of Atg5 (Figs. S2b). Atg5 knock-down enhanced sensitivity to starvation (Fig. S2c) ${ }^{24}$, but did not affect the lifespan of control females under fed conditions (daGS $>$ UAS-Atg5RNAi \pm RU; Fig. 2h). Importantly, the lifespan extension by catalase overexpression was abolished in an Atg5-RNAi background (da-GS $>$ UAS-Atg5RNAi+UAS-cat \pm RU; Fig. 2i). Therefore, autophagy is required for the enhanced longevity by catalase up-regulation.

Redox regulation of autophagy has been described for Atg4 in the context of starvationinduced ROS production in vitro ${ }^{26}$. Atg 4 is the only cysteine peptidase amongst the autophagy components, and is essential for autophagosome biogenesis ${ }^{23}$. Atg4 regulates autophagy by processing Atg8 at two critical stages: 1) the initial cleavage of Atg8, mediated by the redoxinsensitive catalytic cysteine of Atg4, therefore this first step promoting Atg8 lipidation is redox- 
independent; and 2) the subsequent redox-dependent de-lipidation of Atg8, which is selectively inactivated upon oxidation of an adjacent redox-regulatory cysteine in Atg4. Therefore, under oxidising conditions, lipidated Atg8 accumulates due to the redox-mediated suppression of deconjugation by Atg4, thereby enhancing autophagosome biogenesis and promoting Atg4-mediated autophagy (Fig. 3a).

We recently showed that fasting for $24 \mathrm{~h}$ is associated with a strong oxidising shift of bulk cysteine residues in Drosophila in vivo ${ }^{27}$. We therefore hypothesised that the starvation sensitivity of the da-GAL4>UAS-cat females may be attributed to such thiol redox changes. To explore the effects of catalase up-regulation on global thiol redox state, we applied the same redox proteomic technique, OxICAT ${ }^{27}$, to the catalase over-expressing females. In OxICAT, samples undergo differential labelling of cysteine residues according to redox status, followed by trypsin proteolysis and enrichment for cysteine-containing peptides, and finally detection by tandem mass spectrometry. This allows both the identification of redox-responsive cysteine residues, as well as determination of their redox state. The bulk redox state of cysteines in control flies does not change with age, with the majority remaining at $\sim 10-15 \%$ oxidised ${ }^{27}$. In contrast, the catalase overexpressors displayed an oxidising shift in cysteine redox state relative to controls with increasing age (Figs. 3b-d, S3a-d, Table S2). This finding is counter-intuitive, since we are up-regulating an antioxidant enzyme. Therefore, we tested the hypothesis that by quenching $\mathrm{H}_{2} \mathrm{O}_{2}$, catalase overexpression blocks $\mathrm{H}_{2} \mathrm{O}_{2}$ redox signals that up-regulate other antioxidant systems and redox couples. The Keap1/Nrf2 signalling pathway is an appealing candidate for this process, as it is an oxidative stress response pathway that enhances the expression of a range of redox processes and is known to be redox-regulated in Drosophila ${ }^{28}$. To assess this pathway, we used a transgenic reporter for Keap1/Nrf2 activity (gstD-GFP). This pathway was up-regulated with age in controls, but not in the long-lived catalase flies (Fig. S3e), suggesting that catalase over-expression prevented the induction of Keap1/Nrf2 signalling with age and thus the induction of a range of redox processes. Altogether, we have shown that catalase flies undergo an unexpected global oxidising thiol redox shift with age. This oxidation is consistent with the enhancement of autophagy via redox-regulation of Atg4.

The protein sequence of Atg4 is evolutionarily conserved, with both the catalytic cysteine (Cys98 in Drosophila Atg4a) and the adjacent redox-regulatory cysteine (Cys102 in Drosophila Atg4a) present in flies and mammals (Figs. 4a, S4a). To dissect the physiological role of Atg4 redox regulation in vivo, we generated a transgenic knock-in fly line by CRISPR, where the regulatory cysteine in endogenous Atg4a was replaced by a redox-inactive serine residue (C102S mutant). Basal levels of autophagy were not affected under control conditions (Fig. 4b, UAS-cat/+, Atg4aC102S), whereas autophagy induction by catalase over-expression was fully abolished (Fig. 4b, da- 
GAL4>UAS-cat, Atg4a-C102S). Therefore, this redox-regulatory cysteine in Atg4 is required for autophagy induction by redox signalling in vivo, as previously reported in vitro ${ }^{26}$.

To interrogate the role of Atg4a Cys102 in mediating the longevity of the catalase flies, we performed survival assays with the Atg4a-WT CRISPR control line, and reproduced the catalase lifespan extension in this background (Fig. 4c). The Atg4a-C102S point mutation did not affect survival of control flies, confirming that this knock-in alone is not deleterious (Fig. 4d). Critically, in contrast to the Atg4a-WT control, the lifespan extension upon catalase up-regulation was fully abolished in the Atg4a-C102S mutant background (Fig. 4d). Therefore, redox-regulation of autophagy via Atg4a-Cys102 mediates the longevity upon catalase over-expression. Enhancing autophagy is an evolutionarily conserved intervention associated with health and survival benefits, and here we demonstrate that selective redox-mediated up-regulation of autophagy can extend lifespan.

Many attempts have been made to extend lifespan in model organisms by enhancing their antioxidant capacity, notably through the over-expression of antioxidant enzymes, including catalase ${ }^{29-32}$. These trials have been largely unsuccessful, casting doubt on the causative role of ROS and oxidative damage in ageing ${ }^{33}$. Therefore, our finding that catalase over-expression extends lifespan was at first surprising. The original study over-expressing catalase in Drosophila found no effect on lifespan and only modest resistance to oxidative stress by $\mathrm{H}_{2} \mathrm{O}_{2}{ }^{29}$. However, the study used an extra chromosomal copy under its endogenous promoter, resulting in far lower overexpression of catalase ( $\sim 1.75$-fold at the mRNA level and $\sim 1.5$-fold increased enzyme activity). The degree of catalase over-expression is therefore likely to be important for the lifespan extension. Furthermore, the earlier study used only males, while our findings show a robust effect specifically in females. The results of the two studies are therefore not discordant with one another.

Our redox proteomic analysis has revealed that the catalase flies undergo a thiol oxidising shift in bulk cysteine redox state. Interestingly, this pattern is similar to our earlier observations in WT flies under starvation stress ${ }^{27}$. Nutrient deprivation can directly affect redox homeostasis by depleting the provision of important reducing equivalents, such as NADPH and glutathione, mediating an intracellular oxidising shift ${ }^{34}$. Therefore, we suggest that the catalase flies undergo a thiol oxidising shift that is perceived as an internal state of starvation, which triggers the induction of autophagy as a protective response (Fig. 4e). Autophagy plays a fundamental role in healthy physiology, such as cellular differentiation, tissue remodelling, and mitochondrial homeostasis, as well as in the response to stress and the clearance of cellular damage ${ }^{22}$. Consequently, enhanced autophagy is a common denominator of many evolutionary conserved interventions that extend lifespan ${ }^{21,22}$, both genetically, such as down-regulation of insulin signalling 35 , and pharmacologically, for instance rapamycin treatment ${ }^{36}$. Furthermore, direct up-regulation of 
autophagy has been shown to exert health benefits and extend lifespan in a range of model organisms including worms, flies and mice ${ }^{37-39}$. Several components of the autophagy pathway are known to be redox regulated, including $\operatorname{Atg} 3$ and $\operatorname{Atg} 7^{40}$, as well as the focus of our study $\operatorname{Atg} 4^{26}$ Overall, we have shown that shifting the in vivo redox state of Drosophila through over-expression of catalase extends lifespan and healthspan in females through redox regulation of autophagy via a key redox-regulatory cysteine in Atg4a. Our findings further emphasise the importance of finetuning autophagy in health and disease, and demonstrate how manipulation of redox signalling in vivo can ameliorate the effects of ageing. Furthermore, our data are consistent with a growing view in the ageing field that many effects of ROS on longevity are likely to be through alterations in redox signalling rather than through lessening of oxidative damage ${ }^{7,8}$.

\section{Methods}

Fly strains and husbandry. The white Dahomey $\left(w^{D a h}\right)$ strain of Drosophila melanogaster was used as the WT background. The Dahomey stock was collected in 1970 in Dahomey (presently the Republic of Benin), and maintained since then as large population cages, ensuring outbreeding and overlapping generations. The $w^{D a h}$ stock was derived by incorporation of the $w^{1118}$ mutation into the outbred Dahomey background by back-crossing. Flies were either negative ( $\left.w^{\text {Dah }}\right)$ or positive $\left(w^{D a h+}\right)$ for the bacterial cytoplasmic endosymbiont Wolbachia, with infection status confirmed by PCR using published primers against $w s p^{11}$. The $w^{D a h}$ stock was originally achieved by tetracycline treatment of $w^{D a h+11}$. All transgenic lines were back-crossed into the appropriate $w^{D a h}$ background for at least 6-10 generations. See the Supplementary Information for details of all fly strains. Experimental flies were incubated at $25^{\circ} \mathrm{C}$ on a $12 \mathrm{~h}$ light: $12 \mathrm{~h}$ dark cycle with $65 \%$ humidity.

Fly food. Flies were raised on standard sugar-yeast-agar medium (SYA) consisting of: 5\% w/v sucrose (granulated sugar, Tate \& Lyle), 10\% w/v yeast (\#903312, MP Biomedicals), 1.5\% w/v agar (A7002, Sigma), supplemented with nipagin (Sigma H5501; $30 \mathrm{~mL} / \mathrm{L}$ of 10\% w/v nipagin in 95\% EtOH) and propionic acid (Sigma P1386; 0.3\% v/v) as mould inhibitors, added once the food had cooled down to $\sim 60^{\circ} \mathrm{C}^{16}$. Expression via the inducible GeneSwitch system was achieved by addition of the drug RU (RU486/mifepristone; M8048, Sigma) to standard SYA once cooled down to $\sim 60^{\circ} \mathrm{C}$, typically at $200 \mu \mathrm{M}$ from a $0.1 \mathrm{M}$ stock in EtOH. For dietary restriction (DR) experiments, the yeast content was varied to give $1 \%(0.1 \mathrm{x}), 5 \%(0.5 \mathrm{x}), 7.5 \%(0.75 \mathrm{x}), 10 \%(1 \mathrm{x}=$ SYA) or $15 \%(1.5 \mathrm{SYA}) \mathrm{w} / \mathrm{v}$ yeast ${ }^{41}$.

Experimental flies. For all experiments, eggs were collected over a defined period $(<24 \mathrm{~h})$ to ensure a synchronous population and reared at constant density in $200 \mathrm{~mL}$ bottles with SYA ${ }^{41}$. 
232 Eclosing adults of a defined age were kept as a mixed population for $\sim 48 \mathrm{~h}$ to allow mating, then separated into males and females under mild $\mathrm{CO}_{2}$ anaesthesia, and maintained as separate sexes from then on.

Lifespan \& stress assays. Lifespan assays were set up as above, typically with $\mathrm{n} \sim 10-15$ flies per vial and a total of $\mathrm{n} \sim 100-250$ flies per condition. Flies were transferred to fresh food without gassing every $\sim 2-3$ days, with deaths and censors recorded. Stress assays were performed on $\mathrm{d} 7 \mathrm{flies}$ (typically $n>100$ per condition in groups of $\sim 15-20$ flies per vial), with deaths scored regularly following initiation of treatment. See Table $\mathrm{S} 1$ for full survival assay information. For $\mathrm{H}_{2} \mathrm{O}_{2}$ resistance, flies were transferred onto medium containing 5\% v/v $\mathrm{H}_{2} \mathrm{O}_{2}$ (Sigma $\mathrm{H} 1009$ ), 5\% w/v sucrose, $1.5 \% \mathrm{w} / \mathrm{v}$ agar. For paraquat stress, flies were either transferred onto standard SYA food supplemented with $20 \mathrm{mM}$ paraquat (Sigma 856177), or injected with $75 \mathrm{~nL}$ of $1 \mathrm{mg} / \mathrm{mL}$ paraquat in Ringers buffer ( $3 \mathrm{mM} \mathrm{CaCl}_{2}, 182 \mathrm{mM} \mathrm{KCl}, 46 \mathrm{mM} \mathrm{NaCl}, 10 \mathrm{mM}$ Tris base, $\mathrm{pH} 7.2 \mathrm{HCl}$ ) and maintained on standard SYA ${ }^{42}$. Starvation stress was assayed by transferring flies to $1.5 \% \mathrm{w} / \mathrm{v}$ agar medium, which lacks nutrients but allows hydration. Chloroquine (10 mM, Sigma C6628) was prepared in $5 \% \mathrm{w} / \mathrm{v}$ sugar, $1.5 \% \mathrm{w} / \mathrm{v}$ agar. Hyperoxia was performed by incubating flies on standard SYA vials in a glove box chamber set at $90 \% \mathrm{O}_{2}$ using a ProOx controller (BioSpherix). The majority of lifespans (Figs. 1a-b, 2b,h-i, 4c-d, S1d-f, S2c) and stress assays (Figs. 1f-g, 2c,g) were repeated at least twice as independent biological experiments, except Figs. 1h-i and S1c,g,i-m, which were performed once.

Development time. Eggs were collected from flies in cages onto grape juice agar plates over a defined time window $(\sim 4 \mathrm{~h})$. After $\sim 24 \mathrm{~h}$, the resulting L1 larvae were picked onto SYA food at a density of 50 per vial ( $n=500$ total per genotype), and the time to adult eclosion was monitored.

Climbing assay. Climbing ability (negative geotaxis) was assayed essentially as described ${ }^{43}$. Briefly, groups of 15 flies were transferred to a sawn-open $25 \mathrm{~mL}$ serological pipette $(35 \mathrm{~cm}$ long, $1.5 \mathrm{~cm}$ diameter), with the base sealed by parafilm. The flies were tapped down within the column and observed during $45 \mathrm{~s}$, after which their location was recorded. The column was separated into three sections: top $10 \mathrm{~cm}$, middle, bottom $3 \mathrm{~cm}$. Each cohort was evaluated 3 times, using 5 groups

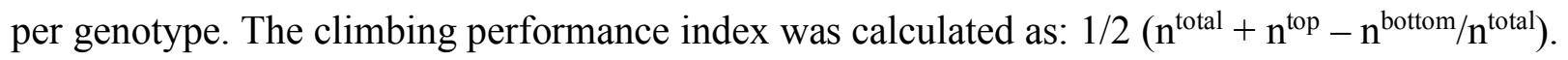

Metabolic and molecular assays. Flies for molecular experiments were rapidly transferred to prechilled microtubes via a small plastic funnel and snap frozen in liquid nitrogen, then stored at $-80^{\circ} \mathrm{C}$ 
circadian variation. For some assays, frozen flies were separated into body segments using forceful manual impact ${ }^{44}$.

Western blotting. Frozen fly samples were homogenised directly into $2 \mathrm{X}$ Laemmli loading buffer (Bio-Rad) supplemented with 5\% v/v $\beta$-mercaptoethanol (Sigma) using a pellet pestle and motor (usually $5 / 10$ females into $100 / 200 \mu \mathrm{L}$ ) and separated by standard SDS-PAGE. The following primary antibodies were used at the indicated dilutions: anti-actin (AbCam Ab1801; 1:1,000), antiAtg8 (a generous gift from K. Köhler ${ }^{45} ; 1: 1,000$ ), anti-catalase (Sigma C0979; 1:10,000), anti-GFP (Cell Signaling \#2955; 1:1,000). Blots were developed using standard ECL, followed by analysis with FIJI (ImageJ) software.

Energy storage assays. Whole body triacylglyceride (TAG) and glycogen levels were measured in $\mathrm{d} 7$ females ( $\mathrm{n}=5$ flies per sample, $\mathrm{n}=6-8$ replicates per genotype) under control (fed) conditions and in response to starvation. For the TAG assay, flies were homogenised in $0.05 \% \mathrm{v} / \mathrm{v}$ Tween-20 and assayed using the Triglyceride Infinity Reagent (Thermo-Scientific TR22421) in a 96-well plate measuring absorbance at $540 \mathrm{~nm}$. For the glycogen assay, flies were homogenised in saturated sodium sulphate, then the subsequent pellet was resuspended in anthrone reagent (Sigma 319899) and assayed in a 96-well plate measuring absorbance at $620 \mathrm{~nm}^{46}$.

OxICAT. To measure the redox state of protein cysteine residues, we performed redox proteomics using OxICAT, with protein isolation, cysteine-residue labelling, peptide preparation and LCMS/MS analysis performed exactly as described previously ${ }^{27}$. See the Supplementary Information for further details.

Statistical analysis. Lifespan and stress assays were plotted as cumulative survival curves, and statistical analysis was performed by Log-Rank test. Other data were analysed by Student's t-test or ANOVA as appropriate in GraphPad Prism 8.

\section{References}

1. Sena, L. A. \& Chandel, N. S. Physiological roles of mitochondrial reactive oxygen species. Mol. Cell 48, 158-167 (2012).

2. Holmstrom, K. M. \& Finkel, T. Cellular mechanisms and physiological consequences of redox-dependent signalling. Nat. Rev. Mol. Cell Biol. 15, 411-421 (2014).

3. Collins, Y. et al. Mitochondrial redox signalling at a glance. J. Cell Sci. 125, 801-806 (2012).

4. Paulsen, C. E. \& Carroll, K. S. Cysteine-mediated redox signaling: chemistry, biology, and tools for discovery. Chem Rev 113, 4633-4679 (2013). 
5. Murphy, M.P.How mitochondria produce reactive oxygen species. Biochem.J. 417, 1-13(2009).

6. Stocker, S., Van Laer, K., Mijuskovic, A. \& Dick, T. P. The Conundrum of Hydrogen Peroxide Signaling and the Emerging Role of Peroxiredoxins as Redox Relay Hubs. Antioxid. Redox Signal. 28, 558-573 (2018).

7. Orr, W. C., Radyuk, S. N. \& Sohal, R. S. Involvement of redox state in the aging of Drosophila melanogaster. Antioxid. Redox Signal. 19, 788-803 (2013).

8. Jones, D. P. Redox theory of aging. Redox Biol. 5, 71-79 (2015).

9. Piper, M. D. W. \& Partridge, L. Drosophila as a model for ageing. Biochim. Biophys. Acta Mol. Basis Dis. 1864, 2707-2717 (2018).

10. Austad, S. N. \& Fischer, K. E. Sex Differences in Lifespan. Cell Metab. 23, 1022-1033 (2016).

11. Toivonen, J. M. et al. No influence of Indy on lifespan in Drosophila after correction for genetic and cytoplasmic background effects. PLOS Genet. 3, e95 (2007).

12. Ikeya, T., Broughton, S., Alic, N., Grandison, R. \& Partridge, L. The endosymbiont Wolbachia increases insulin/IGF-like signalling in Drosophila. Proc. Biol. Sci. 276, 3799-3807 (2009).

13. Lopez-Otin, C., Blasco, M. A., Partridge, L., Serrano, M. \& Kroemer, G. The hallmarks of aging. Cell 153, 1194-1217 (2013).

14. Mair, W., Goymer, P., Pletcher, S. D. \& Partridge, L. Demography of dietary restriction and death in Drosophila. Science 301, 1731-1733 (2003).

15. Fontana, L. \& Partridge, L. Promoting health and longevity through diet: from model organisms to humans. Cell 161, 106-118 (2015).

16. Grandison, R. C., Wong, R., Bass, T. M., Partridge, L. \& Piper, M. D. Effect of a standardised dietary restriction protocol on multiple laboratory strains of Drosophila melanogaster. PLOS One 4, e4067 (2009).

17. Madeo, F., Tavernarakis, N. \& Kroemer, G. Can autophagy promote longevity? Nat. Cell Biol. 12, 842-846 (2010).

18. Rubinsztein, D. C., Marino, G. \& Kroemer, G. Autophagy and aging. Cell 146, 682-695 (2011).

19. Kaushik, S. \& Cuervo, A. M. Proteostasis and aging. Nat. Med. 21, 1406-1415 (2015).

20. Vilchez, D., Saez, I. \& Dillin, A. The role of protein clearance mechanisms in organismal ageing and age-related diseases. Nat. Commun. 5, 5659 (2014).

21. Madeo, F., Zimmermann, A., Maiuri, M. C. \& Kroemer, G. Essential role for autophagy in life span extension. J. Clin. Invest. 125, 85-93 (2015).

22. Hansen, M., Rubinsztein, D. C. \& Walker, D. W. Autophagy as a promoter of longevity: insights from model organisms. Nat. Rev. Mol. Cell Biol. 19, 579-593 (2018).

23. Scherz-Shouval, R. \& Elazar, Z. Regulation of autophagy by ROS: physiology and pathology. Trends Biochem. Sci. 36, 30-38 (2011). 
24. Scott, R. C., Schuldiner, O. \& Neufeld, T. P. Role and regulation of starvation-induced autophagy in the Drosophila fat body. Dev. Cell 7, 167-178 (2004).

25. Kang, C., You, Y. J. \& Avery, L. Dual roles of autophagy in the survival of Caenorhabditis elegans during starvation. Genes Dev. 21, 2161-2171 (2007).

26. Scherz-Shouval, R. et al. Reactive oxygen species are essential for autophagy and specifically regulate the activity of Atg4. EMBO J. 26, 1749-1760 (2007).

27. Menger, K. E. et al. Fasting, but Not Aging, Dramatically Alters the Redox Status of Cysteine Residues on Proteins in Drosophila melanogaster. Cell Rep. 11, 1856-1865 (2015).

28. Sykiotis, G. P. \& Bohmann, D. Keap1/Nrf2 signaling regulates oxidative stress tolerance and lifespan in Drosophila. Dev. Cell 14, 76-85 (2008).

29. Orr, W. C. \& Sohal, R. S. The effects of catalase gene overexpression on life span and resistance to oxidative stress in transgenic Drosophila melanogaster. Arch. Biochem. Biophys. 297, 35-41 (1992).

30. Orr, W. C., Mockett, R. J., Benes, J. J. \& Sohal, R. S. Effects of overexpression of copper-zinc and manganese superoxide dismutases, catalase, and thioredoxin reductase genes on longevity in Drosophila melanogaster. J. Biol. Chem. 278, 26418-26422 (2003).

31. Doonan, R. et al. Against the oxidative damage theory of aging: superoxide dismutases protect against oxidative stress but have little or no effect on life span in Caenorhabditis elegans. Genes Dev 22, 3236-3241 (2008).

32. Perez, V. I. et al. The overexpression of major antioxidant enzymes does not extend the lifespan of mice. Aging Cell 8, 73-75 (2009).

33. Gems, D. \& Doonan, R. Antioxidant defense and aging in C. elegans: is the oxidative damage theory of aging wrong? Cell Cycle 8, 1681-1687 (2009).

34. Filomeni, G., De Zio, D. \& Cecconi, F. Oxidative stress and autophagy: the clash between damage and metabolic needs. Cell Death Differ. 22, 377-388 (2015).

35. Toth, M. L. et al. Longevity pathways converge on autophagy genes to regulate life span in Caenorhabditis elegans. Autophagy 4, 330-338 (2008).

36. Bjedov, I. et al. Mechanisms of life span extension by rapamycin in the fruit fly Drosophila melanogaster. Cell Metab. 11, 35-46 (2010).

37. Simonsen, A. et al. Promoting basal levels of autophagy in the nervous system enhances longevity and oxidant resistance in adult Drosophila. Autophagy 4, 176-184 (2008).

38. Lapierre, L. R. et al. The TFEB orthologue HLH-30 regulates autophagy and modulates longevity in Caenorhabditis elegans. Nat. Commun. 4, 2267 (2013).

39. Pyo, J. O. et al. Overexpression of Atg5 in mice activates autophagy and extends lifespan. Nat. Commun. 4, 2300 (2013). 
40. Frudd, K., Burgoyne, T. \& Burgoyne, J. R. Oxidation of Atg3 and Atg7 mediates inhibition of autophagy. Nat. Commun. 9, 95 (2018).

41. Bass, T. M. et al. Optimization of dietary restriction protocols in Drosophila. J. Gerontol. A Biol. Sci. Med. Sci. 62, 1071-1081 (2007).

42. Kerr, F. et al. Direct Keap1-Nrf2 disruption as a potential therapeutic target for Alzheimer's disease. PLOS Genet. 13, e1006593 (2017).

43. Rogers, I. et al. Ageing increases vulnerability to Aß42 toxicity in Drosophila. PLOS One 7, e40569 (2012).

44. Cochemé, H. M. et al. Using the mitochondria-targeted ratiometric mass spectrometry probe MitoB to measure $\mathrm{H}_{2} \mathrm{O}_{2}$ in living Drosophila. Nat. Protoc. 7, 946-958 (2012).

45. Barth, J. M., Szabad, J., Hafen, E. \& Kohler, K. Autophagy in Drosophila ovaries is induced by starvation and is required for oogenesis. Cell Death Differ. 18, 915-924 (2011).

46. Castillo-Quan, J. I. et al. Lithium Promotes Longevity through GSK3/NRF2-Dependent Hormesis. Cell Rep. 15, 638-650 (2016).

\section{Acknowledgements}

This work was funded by the BBSRC, Wellcome Trust and Max Planck Society to LP. HMC is supported by the Medical Research Council UK (MC-A654-5QB90). Work in the MPM lab is supported by the Medical Research Council UK (MC-U105663142) and by a Wellcome Trust Investigator Award (110159/Z/15/Z). We are grateful to Pirrko Salmiheimo and Mumtaz Ahmad for help with preparation of fly media, Jigna Patel for early technical support, and Mike Harbour and Ian Fearnley for assistance with redox proteomics. We thank members of the Partridge and Cochemé labs for helpful discussions. We acknowledge the Bloomington Drosophila Stock Center and the Vienna Drosophila Resource Center (VRDC) for fly strains, and the BACPAC Resource Center for BAC clones.

\section{Author contributions}

Conceived the project: HMC and LP. Performed experiments: HMC, IB, FC, JICQ and CL. Provided technical support: AF, MB and JA. Generated the CRISPR mutants: SG. Conducted and analysed the redox proteomics: KEM and AMJ. Directed the work: HMC, MPM and LP. Wrote the manuscript: HMC, MPM and LP, with input from all the authors.

\section{Competing interests}

The authors declare no competing interests. 
407

\section{Figure legends}

Fig. 1 | Ubiquitous catalase over-expression extends lifespan in flies, independently of oxidative stress resistance. a, Constitutive, ubiquitous catalase over-expression (da-GAL4>UAScat) extended the survival of female flies in a $w^{\text {Dah }}$ (white Dahomey) WT background relative to the UAS-cat $/+\left(p=5.7 \times 10^{-7}\right)$ and da-GAL4/+ $\left(p=3.3 \times 10^{-6}\right)$ controls. The control lines were not significantly different from each other $(p=0.7504)$. No effect was observed in males $(p>0.05$ for all comparisons). Lifespans were performed with $n=200$ flies per condition. b, Inducible catalase overexpression from early adulthood (d2) using the GeneSwitch system extended the lifespan of female flies (da-GS $>$ UAS-cat \pm RU, $p=1.1 \times 10^{-16}$ ). RU had no effect on the da-GS/+ control line $(p=0.7161)$. Lifespans were performed with $n=225-300$ flies per condition. Inset: catalase overexpression assessed by Western blotting in whole $\mathrm{d} 9$ females (=d7 of RU induction), with actin as a loading control. c, Healthspan, inferred from climbing performance, was improved in catalase over-expressing females. Climbing was assayed on da-GS $>$ UAS-cat females \pm RU to control for effects of eye colour on this behaviour. Data are presented as box-and-whisker plots ( $\mathrm{min} / \mathrm{max}$ error bars) of $n=5$ replicates per condition, each with $n=15$ flies per sample, analysed by unpaired Student's t-test. d, Mortality trajectories of the da-GS>UAS-cat \pm RU survival curves from (b), fitted with a linear regression trendline (dotted line). e, Late onset over-expression of catalase using the inducible GeneSwitch system from either middle-age (d28 and d42) and old-age (d56) extended the lifespan of female flies $\left(\mathrm{p}=7.2 \times 10^{-8}, \mathrm{p}=1.0 \times 10^{-7}\right.$ and $\mathrm{p}=1.4 \times 10^{-3}$, respectively against the $-\mathrm{RU}$ control). Lifespans were performed with $n=270$ flies per condition, and were plotted from point of RU induction relative to the remaining - RU control flies at that age (see Fig. S1g for the original survival data). f, Catalase over-expressing flies were strongly resistant to exogenous $\mathrm{H}_{2} \mathrm{O}_{2}$ stress relative to controls (da-GAL4>UAS-cat $v$. UAS-cat $/+; \mathrm{p}=5.0 \times 10^{-61}$ females, $\mathrm{p}=3.2 \times 10^{-33}$ males). $\mathrm{H}_{2} \mathrm{O}_{2}$ treatment (5\% v/v in sucrose/agar medium) was initiated at $\mathrm{d}$, with $\mathrm{n}=105$ males ( $\mathrm{n}=75$ for da-GS $>$ UAS-cat) and $n=120$ females per condition. $\mathbf{g}$, Catalase over-expressing flies were resistant to chronic dietary paraquat stress relative to control flies (da-GAL4>UAS-cat $v$. UAS-cat/+; $\mathrm{p}=6.93 \times 10^{-31}$ females, $\mathrm{p}=7.99 \times 10^{-16}$ males $)$. Paraquat treatment ( $20 \mathrm{mM}$ in SYA food) was initiated at $\mathrm{d}$, with $\mathrm{n}=100$ flies per condition. $\mathbf{h}$, Catalase over-expressing flies (da-GAL4>UAS-cat) were resistant to acute paraquat stress relative to controls (UAS-cat/+). $\mathrm{d} 7$ females were injected with $75 \mathrm{~nL}$ of $1 \mathrm{mg} / \mathrm{mL}$ paraquat in Ringers buffer (+PQ, $\mathrm{n}=150$ flies) or mock injected with buffer alone (-PQ, $\mathrm{n}=120$ flies). i, Catalase over-expressing flies were resistant to environmental hyperoxia stress relative to controls (da-GAL4 $>$ UAS-cat $v$. UAS-cat/+; $\mathrm{p}=1.7 \times 10^{-8}$ females, $\mathrm{p}=8.1 \times 10^{-3}$ males). Incubation at $90 \% \mathrm{O}_{2}$ was initiated at $\mathrm{d} 7$, with $\mathrm{n}=120$ flies per condition (except $\mathrm{n}=90$ for UAS-cat/+ females). All survival assays (a,b,e,f,g,i) were analysed by Log-Rank test (see Table S1 for full $\mathrm{n}$ numbers and $\mathrm{p}$ values). $\mathrm{n} / \mathrm{s}, \mathrm{p}>0.05 ; *, \mathrm{p}<0.05 ; * *, \mathrm{p}<0.01 ; * * *, \mathrm{p}<0.001$. 
Fig. 2 | Catalase lifespan extension requires autophagy. a, Catalase over-expressor (daGAL4>UAS-cat) and control (UAS-cat/+) females displayed a normal response to dietary restriction (DR). Median lifespan is plotted against the yeast content of the diet, with $1 \mathrm{x}$ corresponding to standard SYA food. Data are the means \pm range of 2 independent lifespan experiments, each set up with $\mathrm{n}=150-160$ flies per genotype, analysed by two-way ANOVA (Fisher's LSD). b, Survival curve on 0.1x-fold yeast from (a). The catalase over-expressor females (da-GAL4>UAS-cat) were longer-lived than controls (UAS-cat/+) under low yeast nutritional conditions $\left(\mathrm{p}=3.6 \times 10^{-10}, \mathrm{n}=160\right.$ flies per genotype). c, Catalase over-expressor females (daGAL4>UAS-cat) were sensitive to starvation stress relative to UAS-cat/+ and da-GAL4/+ controls $\left(\mathrm{p}=5.6 \times 10^{-5}\right.$ and $\mathrm{p}=7.6 \times 10^{-7}$, respectively). No difference was observed in males $(\mathrm{p}>0.05$ for all comparisons). Assays were performed at $\mathrm{d} 7$ with $\mathrm{n}=120$ flies per condition (except $\mathrm{n}=80$ for UAScat/+ females). d-e, Triacylglyceride (TAG, d) and glycogen (e) levels in whole females assayed at $\mathrm{d} 7(\mathrm{t}=0)$ and depletion in response to starvation treatment. Data are presented as box-and-whisker plots ( $\min / \max$ error bars) of $n=4-6$ replicates per genotype, each with $n=5$ females per sample, analysed by unpaired Student's t-test $(p>0.05)$. f, Catalase over-expressor females (daGAL4>UAS-cat) displayed enhanced autophagy induction compared to UAS-cat/+ controls at d7, assessed by Western blotting against Atg8, normalised to actin. Data are means \pm SD of $n=6$ biological replicates, each with $\mathrm{n}=10$ abdomens per sample, analysed by paired Student's t-test ${ }^{*}, \mathrm{p}<0.05$ ). Right, typical bands probed against Atg8 with actin as a loading control (see Fig. S4b for the full blot). g, Catalase over-expressor females (da-GAL4>UAS-cat) were sensitive to treatment with the autophagy inhibitor chloroquine (10 $\mathrm{mM}$ in sucrose/agar medium) relative to UAS-cat $/+$ and da-GAL4/+ controls $\left(\mathrm{p}=2.6 \times 10^{-5}\right.$ and $\mathrm{p}=3.5 \times 10^{-9}$, respectively). Assays were performed at $\mathrm{d} 7$ with $\mathrm{n}=160$ flies per condition. h, Global Atg5 knock-down did not decrease lifespan in a WT background under control conditions (da-GS $>$ UAS-Atg5RNAi $\pm R U ; p=0.4177$ ). Lifespan assays were performed on $n=225-240$ females. $i$, Lifespan extension upon catalase overexpression (da-GS $>$ UAS-cat $\pm \mathrm{RU} ; \mathrm{p}=7.2 \times 10^{-15}$ ) was abolished in an autophagy-deficient background (da-GS $>$ UAS-cat+UAS-Atg5RNAi \pm RU; p=0.1701). Survival assays (b,c,g,h,i) were analysed by Log-Rank test (see Table S1 for full n numbers and $\mathrm{p}$ values). $\mathrm{n} / \mathrm{s}, \mathrm{p}>0.05 ;{ }^{*}, \mathrm{p}<0.05$; $* * *, \mathrm{p}<0.001$.

Fig. 3 | Catalase flies undergo an oxidising shift in global thiol redox state. a, Scheme showing the dual function of Atg4 in autophagy: 1) initial redox-independent cleavage of Atg8, to expose a C-terminal glycine residue enabling lipidation by PE (phosphatidyl-ethanolamine) via the E1-like enzyme Atg7 and the E2-like enzyme Atg3. The conjugated Atg8-PE is involved in autophagosome elongation/closure; 2) redox-dependent de-lipidation of Atg8-PE, allowing interaction and fusion 
477 of the autophagosome with the endosomal-lysosomal compartments, and recycling of cleaved

478 Atg8. Oxidation of a redox-regulatory cysteine selectively inactivates the Atg8-PE de-conjugation 479 activity of Atg4, promoting autophagosome biogenesis and therefore enhancing Atg4-mediated 480 autophagy. b, Redox proteomic (OxICAT) analysis of d7, d28 and d56 catalase over-expressing 481 females (da-GAL4>UAS-cat) compared to control (UAS-cat/+). Distribution of total cysteine 482 residue oxidation levels, plotted as the proportion of the total number of peptides containing unique 483 cysteine residues in each $5 \%$ quantile of percentage oxidation. Data are means \pm SEM of $n=5$ 484 biological replicates. c-d, Oxidation state of cysteine residues present, comparing control versus catalase over-expressor females at d7 (c) and d56 (d). Data points above the diagonal dotted line $($ slope $=1)$ indicate cysteine residues more oxidised upon catalase up-regulation, with red symbols

Fig. 4 | Redox regulation of autophagy via Atg4a Cys102 extends lifespan. a, Multiple sequence alignment of the Atg4a protein from Drosophila with the mouse and human orthologues, showing the catalytic cysteine residue (Cys98 in Drosophila) and the redox-regulatory cysteine (Cys102 in Drosophila). See Fig. S4a for the full sequence. b, Autophagy levels assessed by Western blotting against Atg8. The induction of autophagy in response to catalase over-expression was maintained in Atg4a-WT flies, but abolished in the Atg4a-C102S mutant background. See Fig. S4b for the full blots. Quantification of Atg8 levels by densitometry, normalised to actin as a loading control. Data are means \pm range of $n=2$ biological replicates, analysed by two-way ANOVA (Fisher LSD). c-d, Catalase over-expression extended lifespan in the Atg4a-WT control (c), but not in the redoxinsensitive Atg4a-C102S knock-in background (d). Survival assays (c,d) were analysed by LogRank test (see Table $\mathrm{S} 1$ for full $\mathrm{n}$ numbers and $\mathrm{p}$ values). n/s, $\mathrm{p}>0.05 ; * * *, p<0.001$. e, Scheme 


\section{Figure 1}

a
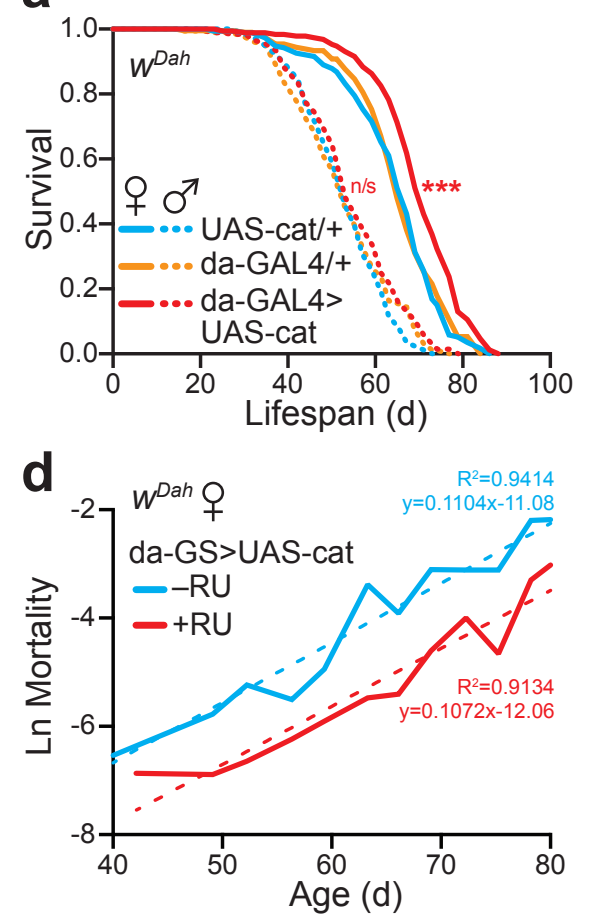

9

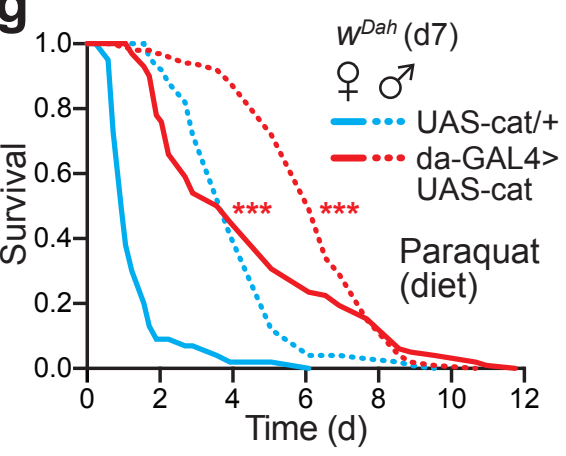

b
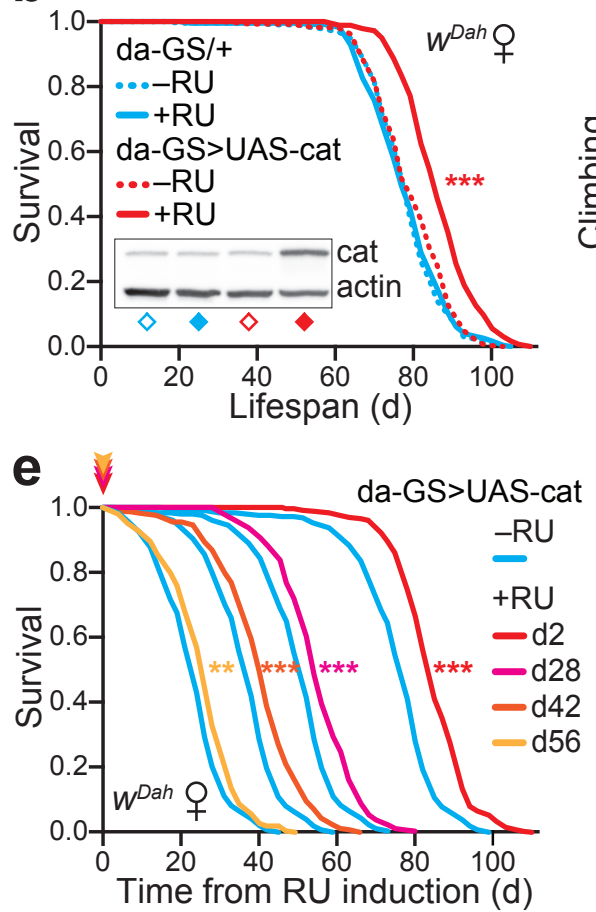

h

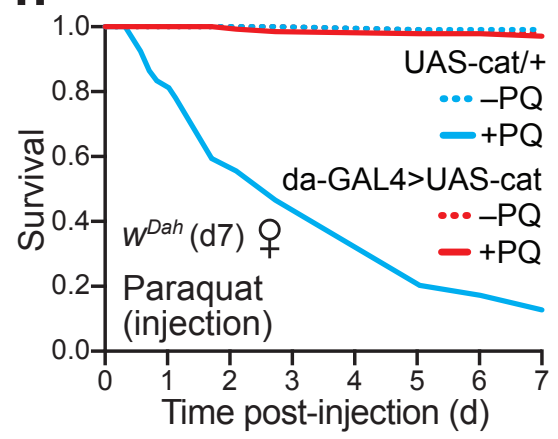

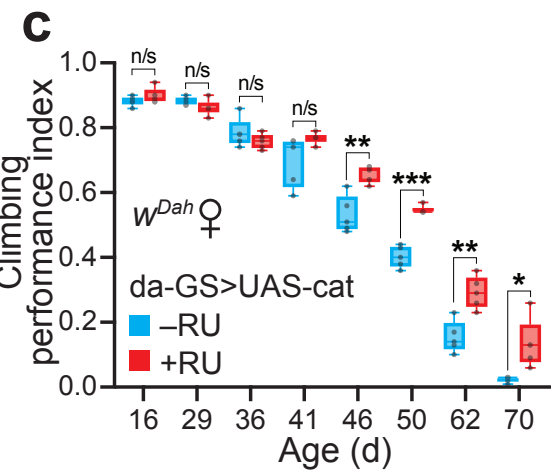

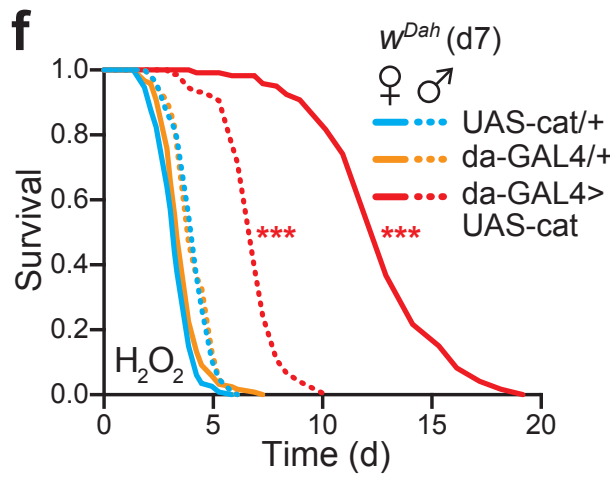

i

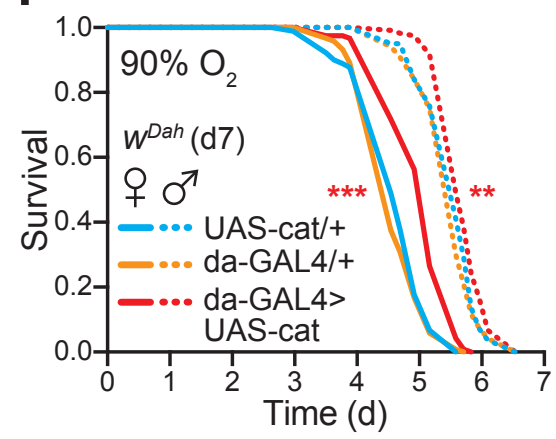




\section{Figure 2}
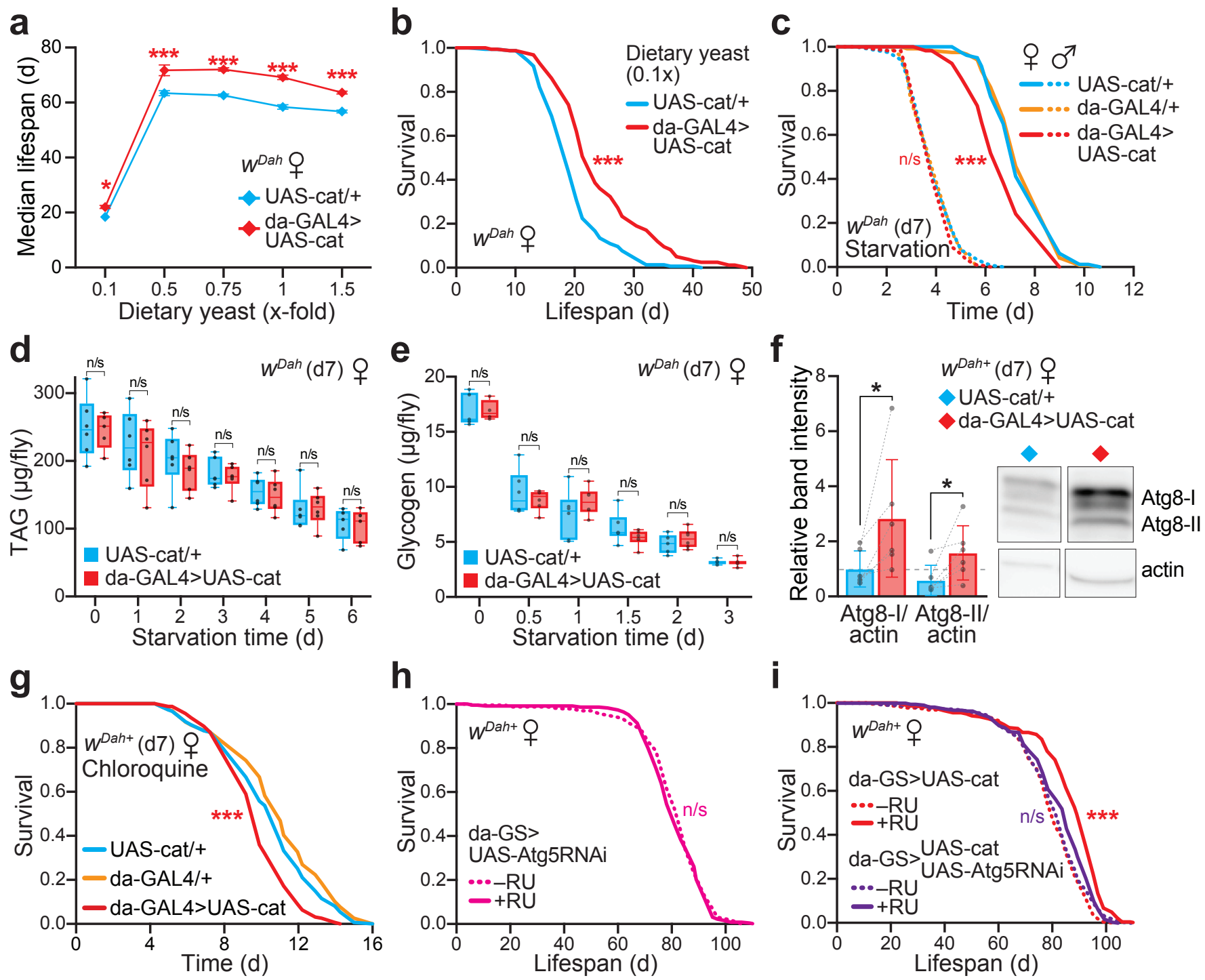


\section{Figure 3}

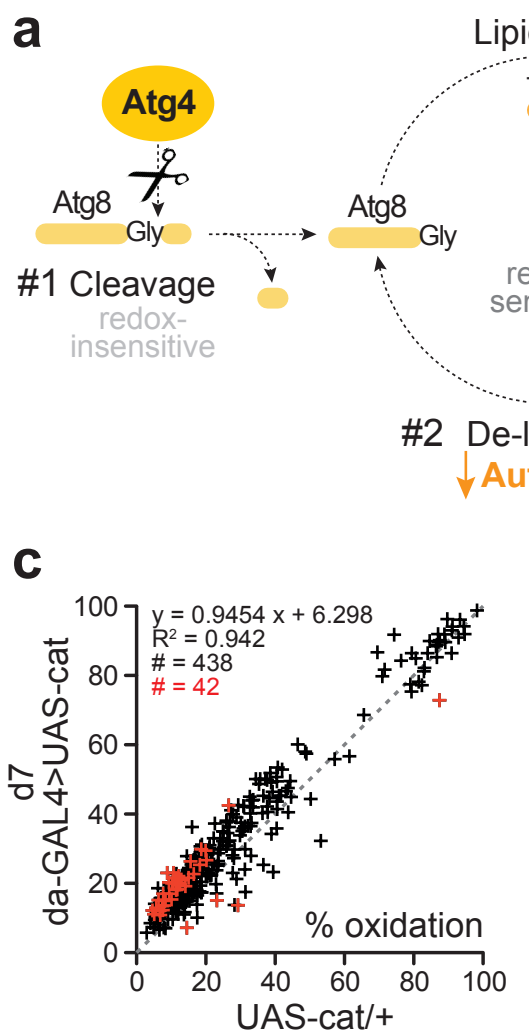

d7
Lipidation

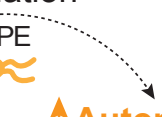

^Autophagy Atg8 Gly

redoxsensitive to

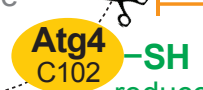
reduced

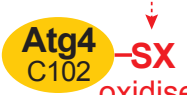
oxidised

e-lipidation

Autophagy

d

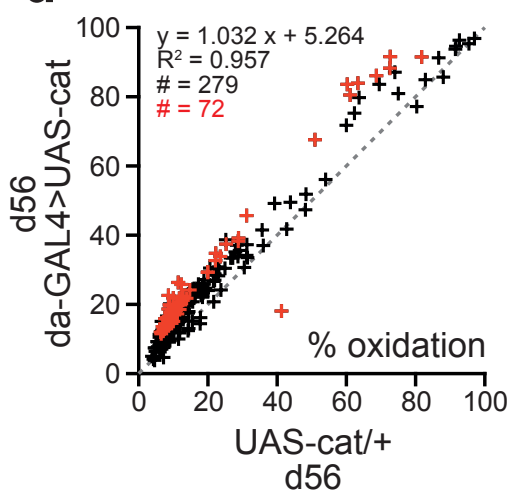

b

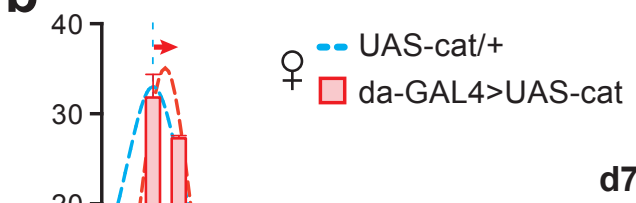

d7

Mean Cys oxidation: $19.6 \%$

$19.9 \%$
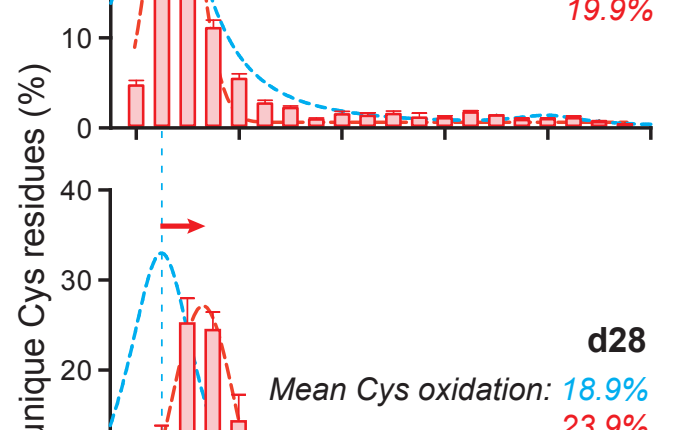

$23.9 \%$

(4)

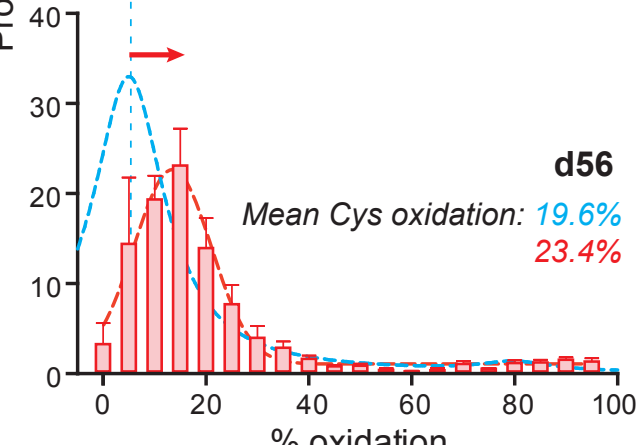




\section{Figure 4}

a

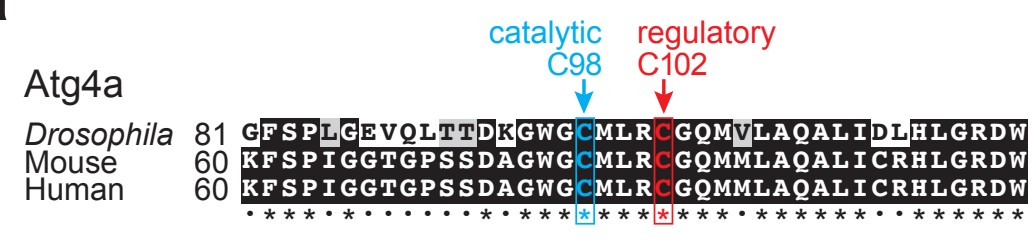

C

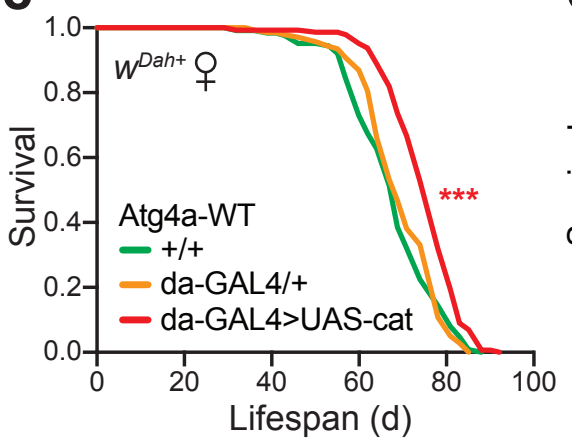

d

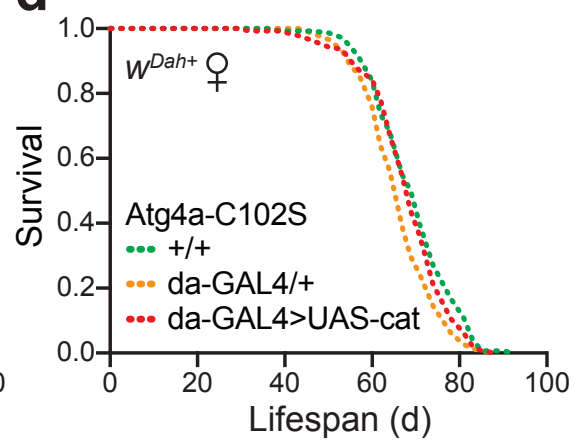

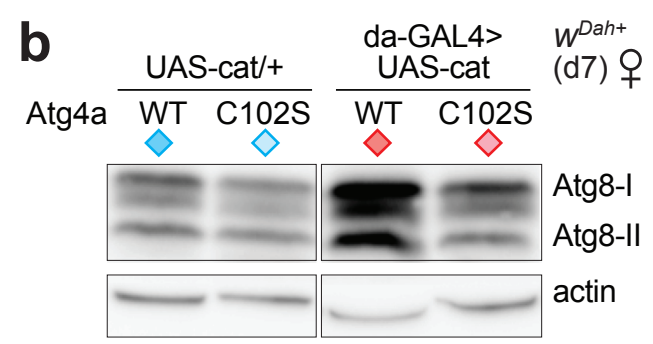

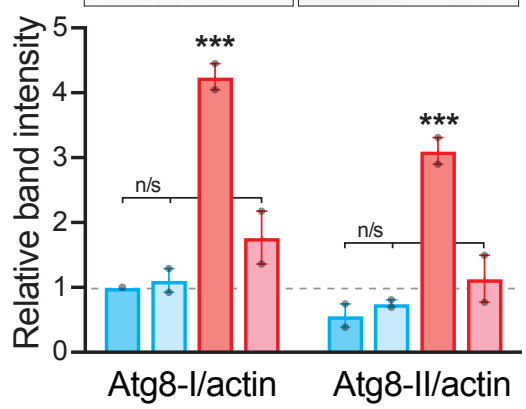

e
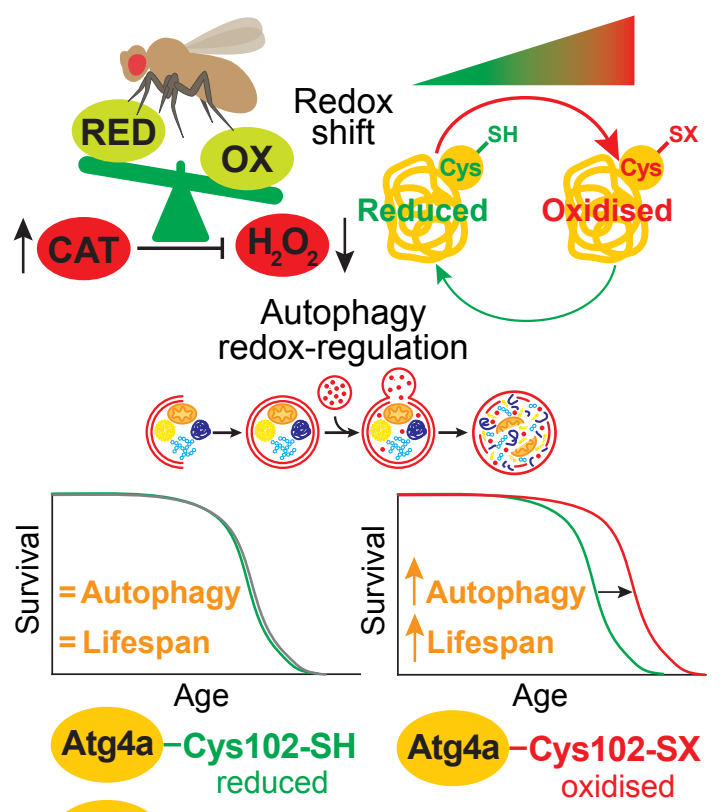

Atg4a-Ser102

redoxinactive 